\title{
Abnormal Expression of Indoleamine 2, 3-Dioxygenase in Human Recurrent Miscarriage
}

\author{
Hongxia Wei, MS ${ }^{\prime *}$, Su Liu, PhD $^{\prime *}$, Ruochun Lian, MMed', \\ Chunyu Huang, MS', Yuye Li, PhD', Lanna Chen, BS', \\ and Yong Zeng, BS
}

\begin{abstract}
Indoleamine 2, 3-dioxygenase (IDO), an immunosuppressive enzyme that mediates the conversion of tryptophan to kynurenine, was shown to play a key role in placental development during normal pregnancy. However, little is known about the pattern of IDO expression in the endometrium and its attendant functional significance in pregnancies complicated with recurrent miscarriage (RM). Immunohistochemical studies of IDO, Foxp3, CD56, and CDI63 expression were performed in endometrial samples from women with RM and healthy fertile controls. Our study found that IDO was localized in glandular epithelial cells, surface epithelial cells, and a small number of cells within the stromal compartment (including stromal cells and leukocytes) in endometrium. Indoleamine 2, 3-dioxygenase expression in the RM group was significantly lower than control group. The Foxp3 and CD56 expression were significantly increased with the elevated IDO expression in controls but not in RM. The percentage of Foxp3+ Tregs was significantly correlated with the level of IDO expression in the control group. Comparatively, no correlation was found between the percentage of CD56 + cells, CDI63 + cells, and the level of IDO expression, no matter in controls and RM patients. This study demonstrated that the downregulation of IDO expression and noncoordinated association between IDO and other endometrial immune cells were associated with RM. Our findings provide insights into the contribution of IDO in immune regulation to maintain normal pregnancy, which could be used to develop potential therapeutic methods for RM.
\end{abstract}

\section{Keywords}

recurrent miscarriage (RM), indoleamine 2, 3-dioxygenase (IDO), forkhead box P3 (Foxp3), CD56, CDI63

\section{Introduction}

Recurrent miscarriage (RM) refers to at least 2 consecutive losses of pregnancy no more than 20 weeks with the same partner, which affects approximately $1 \%$ to $5 \%$ of reproductive-age women. ${ }^{1}$ Although infection, endocrine, metabolic, chromosomal abnormality, anatomic deformation, and autoimmune diseases can result in losses of pregnancy, the exact causes of RM cannot be determined in almost $50 \%$ of cases. ${ }^{2}$ The human endometrium is a highly dynamic tissue that is cyclically shed, repaired, regenerated, and remodeled. A large number of previous studies have shown that endometrium during peri-implantation period plays a pivotal role in embryo implantation and maintenance of normal pregnancy, while its dysfunction may lead to a wide range of reproductive complications such as RM. ${ }^{3,4}$ It has been postulated that the occurrence of RM might be related to immunological dysfunction, ${ }^{5}$ but the precise mechanisms for the disturbance of immune tolerance at the maternal-fetal interface are still poorly understood.
Indoleamine 2, 3-dioxygenase (IDO), a cytoplasmic enzyme that degrades the essential amino acid tryptophan into kynurenine and kynurenic acid, is a key immunomodulatory enzyme that functions to promote immune tolerance by inhibiting T-cell activation and proliferation through tryptophan catabolism. ${ }^{6}$ Initial evidence for IDO-mediated immunosuppression was demonstrated at the maternal-fetal interface. ${ }^{7}$ Inhibition of IDO with 1-methyl-tryptophan could result in rejection of

\footnotetext{
' Shenzhen Key Laboratory of Reproductive Immunology for Periimplantation, Shenzhen Zhongshan Institute for Reproductive Medicine and Genetics, Fertility Center, Shenzhen Zhongshan Urology Hospital, Shenzhen, China

* These authors contributed equally to this work.
}

\section{Corresponding Author:}

Yong Zeng, Shenzhen Key Laboratory of Reproductive Immunology for Periimplantation, Shenzhen Zhongshan Institute for Reproduction and Genetics, Fertility Center, Shenzhen Zhongshan Urology Hospital, No. 100I Fuqiang Road, Futian District, Shenzhen 518045, China.

Email: zengyong1966@gmail.com 
allogeneic fetuses, suggesting that IDO expression at the maternal-fetal interface is necessary to prevent rejection of the fetal allograft. ${ }^{7}$ Subsequent studies have shown that IDO could mediate immunosuppression in autoimmunity, transplantation, and cancer. ${ }^{8,9}$ Various cell types can potentially express IDO at the maternal-fetal interface, including trophoblast cells, decidual immune cells, decidual stromal cells, vascular endothelial cells of chorion and decidua, and epithelial cells. ${ }^{4}$ Ban et al found that the IDO expression level at maternal-fetal interface of $\mathrm{RM}$ is significantly lower than that of normal pregnancy. ${ }^{10}$ Accumulating evidences indicated that reduced activity or expression levels of IDO may contribute to pathological pregnancies. ${ }^{11,12}$ However, the pattern of IDO expression and its attendant functional significance in RM women remain important unknowns.

Regulatory T cells (Tregs) play an important role in tempering immune responses and immune homeostasis. The Tregs levels are significantly decreased in the peripheral blood and decidual tissues of RM patients. ${ }^{13}$ Reduced Tregs counts were closely associated with the onset of unexplained RM. ${ }^{14}$ Previous studies have shown that IDO pathway contributes to regulation of Foxp $3^{+}$Tregs commitment and function. In vitro, tryptophan deprivation acts synergistically with kynurenine metabolites to promote de novo Foxp $3^{+}$Treg differentiation and activation of suppressor activity in Tregs. ${ }^{15-18}$ In vivo, IDO inhibitors or genetic ablation of IDO1 genes prevents antigen-specific Tregs differentiation in response to mucosal antigen challenge. ${ }^{19,20}$ However, it has not been reported the relationship between endometrial IDO expression and Tregs in RM patients.

Natural killer (NK) cells play a role in inhibiting the growth of several types of tumors. ${ }^{21}$ Tryptophan-derived catabolic kynurenine can reduce NK cell number and inhibit the activation of NK cells by downregulating surface recognition receptors NKp46 and NKG2D. ${ }^{22}$ Natural killer cells are the most abundant leukocyte population in decidua and likely play an important role in the establishment and maintenance of normal pregnancy. ${ }^{23,24}$ Although inhibited effects of IDO on NK cell accumulation and cytotoxic activity have been reported in tumor progression, ${ }^{22,25,26}$ little is known about the relationship between human endometrial IDO expression and NK cells in pregnancies complicated with RM.

Macrophages, which can be divided into classically activated (M1) and alternatively activated (M2) phenotype, play a vital role in immune response. Generally, M1-type macrophages can defend the body against the pathogens and tumor cells, while M2-type macrophages are considered to suppress inflammatory responses and adaptive immunity and stimulate angiogenesis and tissue remodeling, ${ }^{27,28}$ The CD163, a scavenger receptor, is expressed exclusively on the surface of monocytes and macrophages ${ }^{29}$ and is a marker of M2 macrophages. ${ }^{30,31}$ Previous studies showed that IDO induce the differentiation of macrophages into M2-type rather than M1-type macrophages. ${ }^{32}$ However, it is still unclear about the relationship between IDO and M2-type macrophages at the maternalfetal interface.
Taking into account the particular immunological situation of the endometrium, it was considered worthwhile to investigate levels of IDO expression and the percentages of Foxp $3^{+}$ Tregs, CD $56^{+} \mathrm{NK}$ cells, and $\mathrm{CD} 163^{+} \mathrm{M} 2$-type macrophages in the endometrium during peri-implantation period. The current study aimed to localize IDO in the endometrium, investigate IDO expression between patients associated with RM and healthy fertile controls, and undertake a correlation study on IDO and other immune cells to determine the role of IDO in the immune regulation at the maternal-fetal interface.

\section{Materials and Methods}

\section{Study Population}

Women who visited Shenzhen Zhongshan Urology Hospital from January 2017 to December 2017 were consecutively enrolled. Of those 49 normal fertile controls and 58 patients with RM were recruited. All patients aged younger than 40 and have a regular menstrual cycle from 26 days to 35 days and were in mid-luteal phase confirmed by hematoxylin and eosin (H\&E) staining. Recurrent miscarriage was defined as at least 2 consecutive clinically spontaneous abortions that occurred in no more than 20 weeks of gestation. Patients who previously or currently had autoimmune or thyroid-related disease, hydrosalpinx, uterine malformation confirmed by ultrasonographic and abnormal karyotypes, and chronic endometritis confirmed by CD138 staining were excluded from RM. Women who had a live-birth baby after their first in-vitro fertilization-embryo transfer cycle due to male factors were defined as the control group. None of the included patients had received any medical treatment before endometrial biopsy. Written informed consent was obtained from individual patients for the use of the endometrial specimens. This study was approved by the ethics committee of Shenzhen Zhongshan Urology Hospital.

\section{Immunohistochemical and Imaging Analyses}

Endometrial tissues were collected during the mid-luteal phase using an endometrial curette. The endometrial samples were fixed with $4 \%$ paraformaldehyde 6 to 12 hours at room temperature after removing blood and washed with phosphatebuffered saline (PBS). Then, all samples were processed into paraffin within 48 hours. Paraffin-embedded endometrial tissues were cut into $4-\mu \mathrm{m}$ sections followed by deparaffinization and dehydration for immunohistochemistry. One section was selected for H\&E staining to confirm the histologic phase. The endogenous peroxidase activity was blocked by $3 \%$ hydrogen peroxide and the nonspecific binding was blocked by $5 \%$ bovine serum albumin for 20 minutes. Then, the sections were incubated with primary antibodies targeted to IDO (Cell Signaling Technology, Danvers, Massachusetts), Foxp3 (eBioscience, San Diego, California), CD56 (Gene Tech Company, Shanghai, China), and CD163 (Novocastra, Tokyo, Japan) at $37^{\circ} \mathrm{C}$ for 1 hour followed by washing with PBS 3 times and PBS $+0.1 \%$ Tween 20 (PBST) for 3 minutes. Details 
Table I. Antibodies Table. ${ }^{a}$

\begin{tabular}{lllc}
\hline Antibody & Manufacturer & Catalog Number & Dilution \\
\hline Anti-IDO & Cell Signaling Technology & 86630 & $1: 300$ \\
Anti-Foxp3 & eBioscience & I4-4777 & $1: 100$ \\
Anti-CD56 & Gene Tech & GT200529 & $1: 400$ \\
Anti-CDI63 & Novocastra & NCL-L-CDI63 & $1: 200$ \\
\hline
\end{tabular}

Abbreviation: IDO, indoleamine 2, 3-dioxygenase.

${ }^{\mathrm{a}}$ Details of sources and concentrations of antibodies used for immunohistochemistry staining.

of all antibodies used in the study were listed in Table 1. The sections were then incubated with secondary antibodies (Dako Cytomation, Glostrup, Denmark) for 30 minutes, followed by washing with PBS 3 times. Antibodies binding were detected with a brown precipitate after stained with peroxidase substrate 3, 3-diaminobenzidine (DAB; Dako Cytomation, Glostrup, Denmark) and counterstained with hematoxylin to allow visualization of the nuclei and dehydrated. Finally, quantification of immunohistochemistry data was analyzed by the Vectra automated quantitative pathology imaging system (Perkin Elmer, Waltham, Massachusetts). Only the images filled with over $80 \%$ of tissues were included in the analysis. The percentages of Foxp $3^{+}$Tregs, CD56 ${ }^{+}$NK cells, CD163 ${ }^{+}$M2-type macrophages were calculated as the number of DAB-positive cells to the total number of endometrial cells at a magnification of $200 \times$ field $\left(0.95 \mathrm{~mm}^{2}\right)$ by 2 independent observers. Thirty fields were captured totally for one sample. For IDO, the staining was graded by 2 observers independently based on both the staining intensity and positivity. Grade 1, no cells were stained or stained weakly and scarcely positive; grade 2 , the cells were stained moderately and focally positive; grade 3 , the cells were stained strongly and diffusely positive. ${ }^{10,33}$

\section{Statistical Analysis}

Statistical analysis was performed using SPSS version 20.0 (SPSS Inc, Chicago, Illinois). The distribution of the data was examined prior to analysis. The data were presented as mean (SD; normal distributed data). Statistical comparisons between the groups were made by Student $t$ test (normal distributed data) or Mann-Whitney $U$ test (abnormally distributed data). One-way ANOVA test was used for multiple group comparisons (normal distributed data). The correlations between the variables in the endometrium were performed by Pearson correlation. Differences were considered to be significant at $P<.05$.

\section{Results}

\section{Clinical Characteristics of the Study Population}

The characteristics of the study population are shown in Table 2. There were no significant differences in maternal age, prepregnancy body mass index, and baseline endocrine data including follicle-stimulating hormone, luteinizing hormone,
Table 2. Baseline and Cycle Characteristics of Control Women and RM Patients. ${ }^{a}$

\begin{tabular}{lccc}
\hline Characteristics & Control $(\mathrm{n}=49)$ & $\mathrm{RM}(\mathrm{n}=58)$ & $P$ Value \\
\hline Maternal age (year) & $31.10(3.56)$ & $32.36(3.05)$ & .054 \\
Prepregnancy BMI $\left(\mathrm{kg} / \mathrm{m}^{2}\right)$ & $20.79(2.29)$ & $21.44(3.44)$ & .626 \\
Basal FSH $(\mathrm{mlU} / \mathrm{mL})$ & $7.15(1.94)$ & $6.39(2.12)$ & .133 \\
Basal LH $(\mathrm{mlU} / \mathrm{mL})$ & $4.93(1.90)$ & $4.62(1.94)$ & .510 \\
Basal E2 $(\mathrm{pg} / \mathrm{mL})$ & $33.57(9.13)$ & $28.96(10.61)$ & .079 \\
Basal PRL $(\mathrm{ng} / \mathrm{mL})$ & $17.67(9.46)$ & $16.18(8.06)$ & .451 \\
Basal P $(\mathrm{ng} / \mathrm{mL})$ & $0.53(0.19)$ & $0.44(0.19)$ & .073 \\
FT3 $(\mathrm{pg} / \mathrm{mL})$ & $3.01(0.33)$ & $2.86(0.66)$ & .403 \\
FT4 $(\mathrm{ng} / \mathrm{mL})$ & $1.30(0.66)$ & $1.24(0.16)$ & .197 \\
TSH $(\mu l \mathrm{ll} / \mathrm{mL})$ & $2.06(0.98)$ & $2.23(1.18)$ & .563 \\
\hline
\end{tabular}

Abbreviations: $\mathrm{BMI}$, body mass index; $\mathrm{E} 2$, estrodiol; $\mathrm{FSH}$, follicle-stimulating hormone; FT3, free triiodothyronine; FT4, free thyroxine; LH, luteinizing hormone; P, progesterone; PRL, prolactin; RM, recurrent miscarriage; TSH, thyroid-stimulating hormone.

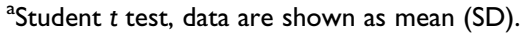

estrodiol, prolactin, free triiodothyronine, free thyroxine, and thyroid-stimulating hormone, between control group and RM group.

\section{Immunohistochemical Grading of the Expression of IDO in Endometrium and Comparison of IDO Between Control and RM}

First, we examined the localization of IDO in the endometrium by immunohistochemical staining. Indoleamine 2, 3-dioxygenase was mainly localized in glandular epithelial cells, surface epithelial cells and a small number of cells within the stromal compartment (including stromal cells and leukocytes) in endometrium (Figure 1A-C), which is consistent with previous studies. ${ }^{34,35}$ We used the immunostaining grading scale (Figure 1A-C) to evaluate the expression levels of IDO in control and RM group. The grading was performed based on staining intensity and positivity, as described in materials and methods. Using this grading scale, we compared IDO expression between the control women and the RM patients. Indoleamine 2, 3-dioxygenase expression in the RM patients was significantly lower than in the control women $(P=.001$; Figure 1D). Our results demonstrated that the downregulation of IDO expression in the endometrium was associated with RM, suggesting that IDO might be involved in the pathophysiology of RM.

\section{Immunohistochemical Examination of Foxp3 in Endometrium and the Relationship Analysis Between IDO and Foxp $3^{+}$Tregs}

To clarify the pathophysiological function of IDO expression in endometrium, we investigated the expression of Foxp3, CD56, and CD163 in endometrium by immunohistochemical staining. We found that Foxp3 localized in endometrial stromal cells with nuclear patterns in both control women and RM patients (Figure 2A and B), which is different from IDO. Then, 


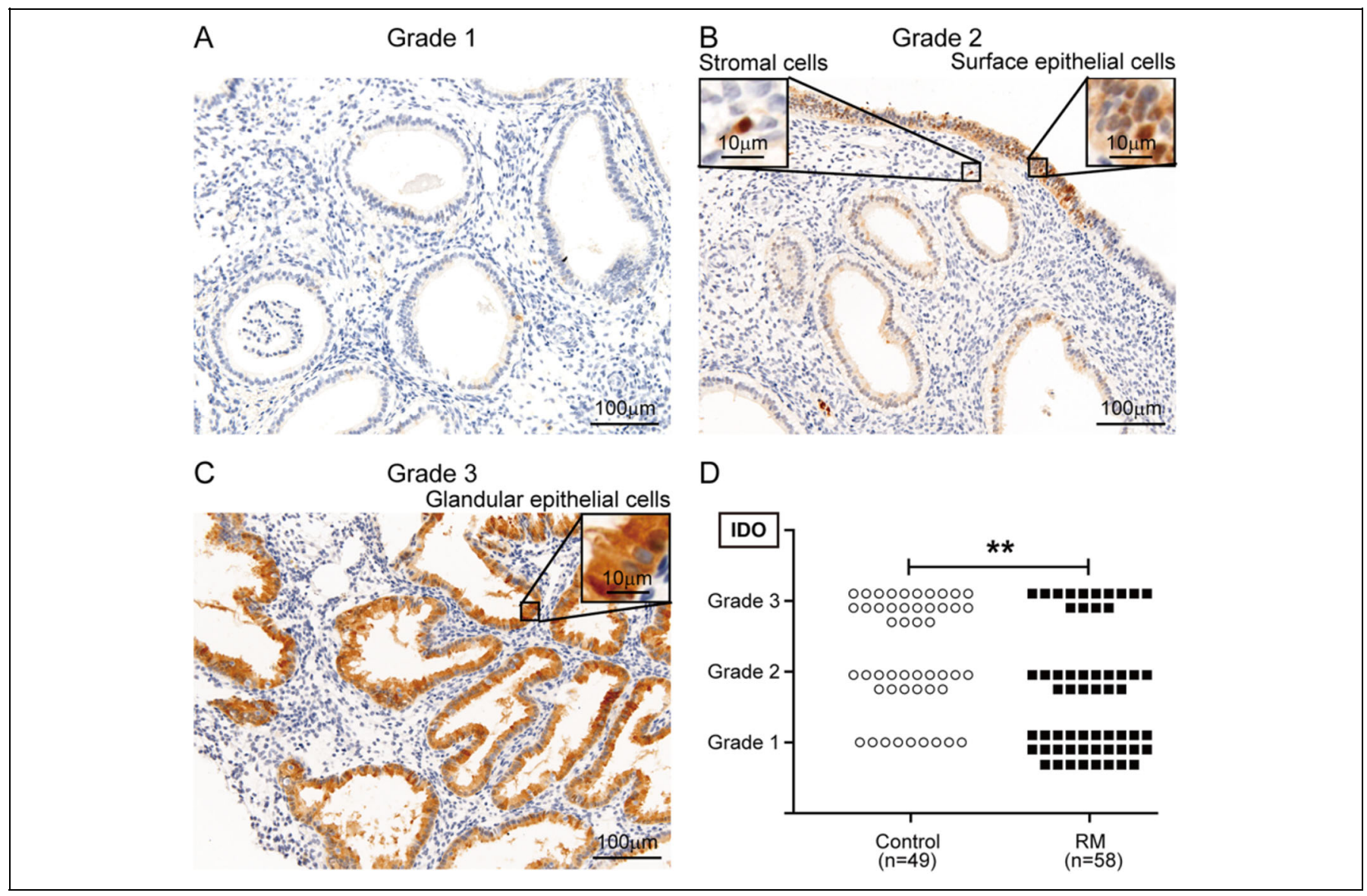

Figure I. Immunohistochemical grading of the expression of IDO in endometrium and comparison of IDO between control and RM. The grading was performed based on staining intensity and positivity, as described in the materials and methods, grade I: no or weak staining and scarcely positive (A); grade 2: moderately stained and focally positive, IDO localized in surface epithelial cells and the stromal compartment (B); grade 3: strongly stained and diffusely positive, IDO localized in glandular epithelial cells (C); the statistical chart showing the expression level of IDO in endometrium between the control group $(n=49)$ and the RM group $(n=58)(D) . * * P<.01$, Mann-Whitney $U$ test; scale bars $=I 00 \mu m$, with insets $=10 \mu \mathrm{m}$. IDO indicates indoleamine 2, 3-dioxygenase; RM, recurrent miscarriage.

we divided both control women and RM patients into 3 subgroups according to the level of IDO expression. We observed a significant elevation of Foxp $3^{+}$Tregs with the increased IDO expression in the control women $(0.06 \pm 0.02 \%, \mathrm{n}=9$ vs 0.08 $\pm 0.04 \%, \mathrm{n}=16$ vs $0.09 \pm 0.05 \%, \mathrm{n}=24, P=.028$ Figure $2 \mathrm{C})$, but not in RM $(0.09 \pm 0.04 \%, \mathrm{n}=28$ vs $0.11 \pm$ $0.05 \%, \mathrm{n}=16$ vs $0.10 \pm 0.04 \%, \mathrm{n}=14, P=.219$; Figure 2D). The percentage of Foxp3 $3^{+}$Tregs was significantly correlated with the level of IDO expression in the control group $(r=$ $0.379, P=.007 ;$ Figure $2 \mathrm{E}$ ). In contrast, no correlation was found between the percentage of Foxp $3^{+}$Tregs and the level of IDO expression in the RM group $(r=.188, P=.176$; Figure $2 \mathrm{~F})$.

\section{Immunohistochemical Examination of CD56 in Endometrium and the Relationship Analysis Between IDO and CD56 ${ }^{+}$NK Cells}

To investigate the association between IDO and NK cells in the endometrium, the expression of CD56 in control group and RM group was also examined by immunohistochemical staining. The CD56 $6^{+} \mathrm{NK}$ cells were membrane stained and found distributed in the endometrium of both control group and RM group (Figure 3A and B). The expression of CD56 in subgroup of grade 3 (scaling IDO expression level) was significantly higher than that in subgroup of grade 2 under control group $(6.97 \pm 4.13 \%, \mathrm{n}=16$ vs $11.91 \pm 6.79 \%, \mathrm{n}=24, P=.029$; Figure 3C). In contrast, there was no significant difference of CD56 expression among 3 subgroups in the RM group (8.58 \pm $5.35 \%, \mathrm{n}=28$ vs $7.57 \pm 6.49 \%, \mathrm{n}=16$ vs $11.92 \pm 7.20 \%, \mathrm{n}$ $=14, P=.497$; Figure 3D). The comparable expression of CD56 in subgroups of grade 1 and grade 2 in control women may be due to insufficient specimens. However, the percentage of $\mathrm{CD} 56^{+} \mathrm{NK}$ cells was not correlated with the level of IDO expression in both control group $(r=0.246, P=.088$; Figure $3 \mathrm{E})$ and RM group $(r=0.162, P=.225$; Figure $3 \mathrm{~F})$.

\section{Immunohistochemical Examination of CD/63 in Endometrium and the Relationship Analysis} Between IDO and CDI63+ M2-type Macrophages

We also examined CD163 expression in endometrium by immunohistochemical staining. Similar to CD56, CD163 ${ }^{+}$ 


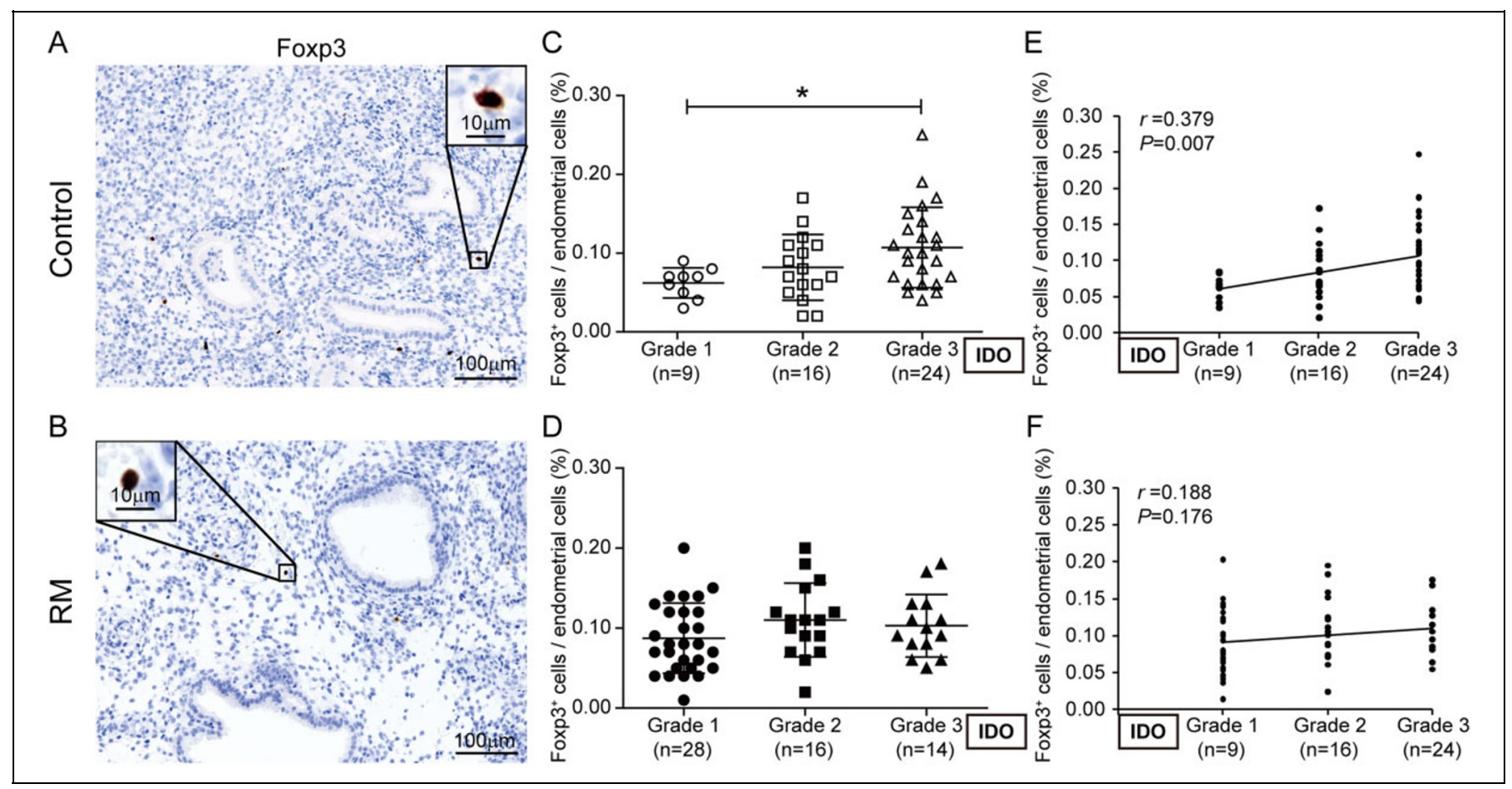

Figure 2. Immunohistochemical examination of Foxp3 in endometrium and the relationship analysis between IDO and Foxp $3^{+}$Tregs. Representative images taken at a magnification of $200 \times$ field of endometrial Foxp $3^{+}$Tregs in control women (A) and RM patients (B). Scale bars $=100 \mu \mathrm{m}$, with insets $=10 \mu \mathrm{m}$. Summary graph of statistical dot plot (mean [SD]) showing the percentages of endometrial Foxp3 among 3 subgroups in control women (grade $I, n=9$; grade $2, n=16$; grade $3, n=24 ; C$ ) and RM patients (grade I, $n=34 ;$ grade 2 , $n=18$; grade 3 , $\mathrm{n}=16$; D). $* P<.05$, one-way analysis of variance test. Numerical correlation between the percentage of Foxp $3^{+}$Tregs and the level of IDO expression in healthy fertile controls (E) and patients with RM (F). Pearson correlation, $r$ and $P$ values as indicated. IDO indicates indoleamine 2 , 3-dioxygenase; RM, recurrent miscarriage.

cells were membrane stained and found distributed in the endometrium of both control group and RM group (Figure 4A and B). There were no significant differences of the percentage of $\mathrm{CD}_{163^{+}}$cell in the endometrium among 3 subgroups of both control group $(2.00 \pm 1.41 \%, \mathrm{n}=9$ vs $1.82 \pm 0.76 \%, \mathrm{n}=16$ vs $1.82 \pm 0.69 \%, \mathrm{n}=24, P=.854)$ and RM group ( $2.09 \pm$ $0.89 \%, \mathrm{n}=28$ vs $2.11 \pm 1.06 \%, \mathrm{n}=16$ vs $2.28 \pm 1.26 \%$, $\mathrm{n}=14, P=.849$; Figure $4 \mathrm{C}$ and $\mathrm{D})$. No correlation was found between the percentage of $\mathrm{CD} 163^{+}$cell and the level of IDO expression in both control group $(r=-0.066, P=.650$; Figure 4E) and RM group ( $r=0.070, P=.604$; Figure $4 \mathrm{~F})$.

\section{Discussion}

Endometrium, serving as an immunologically privileged tissue, plays an essential role as sensor of embryo quality and driver of pregnancy success. ${ }^{4,36}$ Indoleamine 2, 3-dioxygenase is widely distributed at the maternal-fetal interface and plays a vital role in inducing immune tolerance by providing a low tryptophan environment. ${ }^{34,37,38}$ There have been accumulating evidences showed decreased levels of IDO in the placenta and decidua from RM, suggesting an important role for IDO in the maintenance of normal pregnancy. ${ }^{10,33}$ Our current study described the precise distribution of IDO in endometrium. Indoleamine 2, 3-dioxygenase was localized in glandular epithelial cells, surface epithelial cells, and a small number of cells within the stromal compartment (including stromal cells and leukocytes) of endometrium. In consistent with previous studies, ${ }^{10}$ our data showed that the expression level of IDO was downregulated in RM patients compared to controls. The interferon (IFN)- $\gamma$ is an important and potent stimulator of IDO expression. ${ }^{39}$ The production of IFN- $\gamma$ by decidual and peripheral blood mononuclear cells is found to be decreased in RM, which may lead to reduced IDO expression and activity. ${ }^{39}$ It is possible that reduced levels of IDO protein may contribute to disorders of pregnancy such as RM.

There are many immune cells in endometrium playing positive roles in maintaining maternal immune tolerance. The Tregs have been suggested to be necessary for the maintenance of maternal-fetal tolerance, and there are compelling evidences showing that Foxp3 serves as a lineage specification factor for Tregs. ${ }^{40,41}$

In this study, the expression of Foxp3 in endometrium increased significantly with the increasing IDO expression in control women, but not in RM patients. There was a statistically significant correlation between Foxp3 and IDO expression in control women. Previous studies revealed that IDO could modify immune responses in 2 ways: by consuming tryptophan to trigger amino-acid-sensing signal transduction pathways; and by producing kynurenine, a natural ligand for 


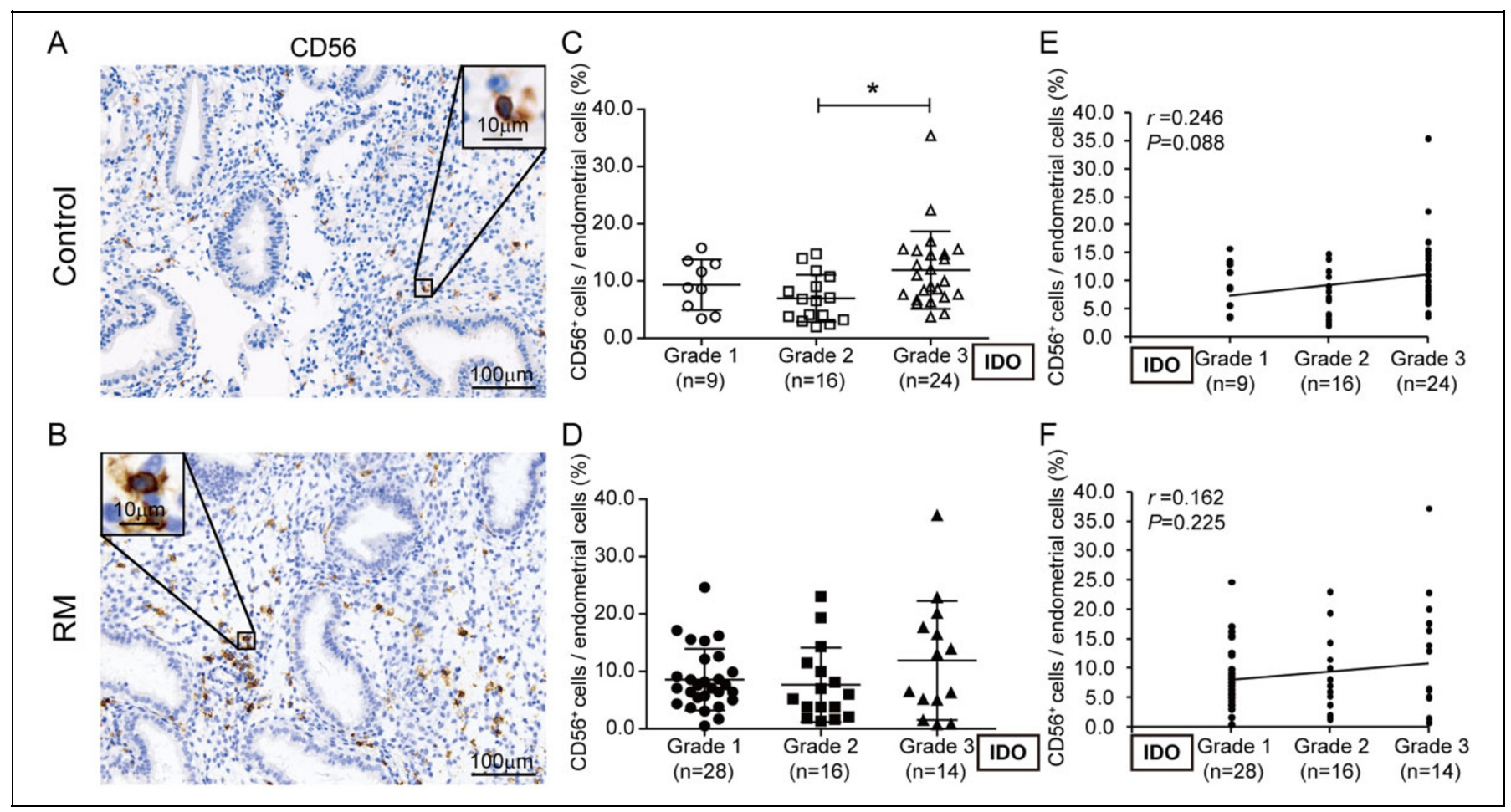

Figure 3. Immunohistochemical examination of CD56 in endometrium and the relationship analysis between IDO and CD56 ${ }^{+} \mathrm{NK}_{\text {cells. }}$ Representative images taken at a magnification of $200 \times$ field of endometrial CD56 ${ }^{+} \mathrm{NK}$ cells in control women (A) and RM patients (B). Scale bars $=100 \mu \mathrm{m}$, with insets $=10 \mu \mathrm{m}$. Summary graph of statistical dot plot (mean [SD]) showing the percentages of endometrial CD56 among 3 subgroups in control group (grade I, $n=9$; grade $2, n=16$; grade $3, n=24 ; C$ ) and RM group (grade I, $n=28 ;$ grade 2, $n=16$; grade 3, $n=14$; D). $* P<.05$, one-way analysis of variance test. Numerical correlation between the percentage of CD56 ${ }^{+} \mathrm{NK}$ cells and the level of IDO expression in healthy fertile controls $(E)$ and patients with RM (F). Pearson correlation, $r$ and $P$ values as indicated. IDO indicates indoleamine 2 , 3-dioxygenase; NK, natural killer; RM, recurrent miscarriage.

the aryl hydrocarbon receptor (AhR). ${ }^{42}$ Insufficiency of tryptophan can activate GCN2 kinase activity, which could block T helper 17 differentiation ${ }^{43,44}$ and promote Treg cell differentiation and activation of suppressor activity in mature Tregs. ${ }^{15,16}$ In the case of kynurenine-pathway metabolites, the effect on AhR appears to be immunosuppressive and also promotes differentiation of Foxp $3^{+}$Tregs. ${ }^{45,46}$ Taken together with our data, we postulate that IDO expressed at the maternal-fetal interface may generate a low tryptophan environment, which subsequently inhibit T-cell proliferation or induce Tregs, ${ }^{15,47}$ but the interaction between IDO and Treg cells was impaired in patients with RM.

Natural killer cells are mainly identified by the surface expression levels of CD56 and CD16, and uterine NK cells show a prevalence of the $\mathrm{CD} 56^{+} \mathrm{NK}$ cell compartment, which have a different phenotype from peripheral blood NK cells. ${ }^{48}$ In vitro, IDO could prevent NK cell accumulation through inducing cell death ${ }^{25,26}$ and prevent NK cell activation by downregulating surface recognition receptors NKp46 and NKG2D. ${ }^{22}$ However, there are few studies on the immunoregulatory effect of IDO on NK cells in the endometrium. In this study, CD56 expression was found significantly increased with the elevated IDO expression in control women but not in RM patients. However, there was no correlation between CD56 and IDO expression in both control women and RM patients. Actually,
NK cells are not a homogeneous cell population and the diversity of NK cell receptors and functions have been demonstrated. The cellular interactions and local microenvironment could provide important signals to shape NK cell properties. ${ }^{49}$ Thus, further studies are necessary to clarify the phenotype of the increasing endometrial NK cells corresponding with the elevated IDO expression in normal controls. Taken together, although we cannot conclude the immunoregulatory role of IDO on the endometrial NK cells, the results imply the noncoordinated expression of CD56 and IDO may lead to RM.

Macrophages can be divided into the M1 macrophages and the M2 macrophages. ${ }^{50}$ Alternatively activated macrophages are distinguished from M1 macrophages by the expression of CD163 and production of immunosuppressive cytokines. ${ }^{51}$ Macrophages can switch from an activated M1 state to M2, and vice versa, depending on the environmental cytokines they are exposed. ${ }^{52}$ In our study, the expression of CD163 in the endometrium was not affected by IDO expression, no matter from normal fertile controls or RM patients. No correlation was found between CD163 and IDO expression in both normal controls and RM patients. Previous studies showed contradictory conclusions on the role of IDO in macrophage differentiation. There are evidences showing that IFN- $\gamma$ could upregulate IDO expression in the murine and human dendritic cells ${ }^{39}$ and monocytes/macrophages. ${ }^{52,53}$ Accompanied by a certain 


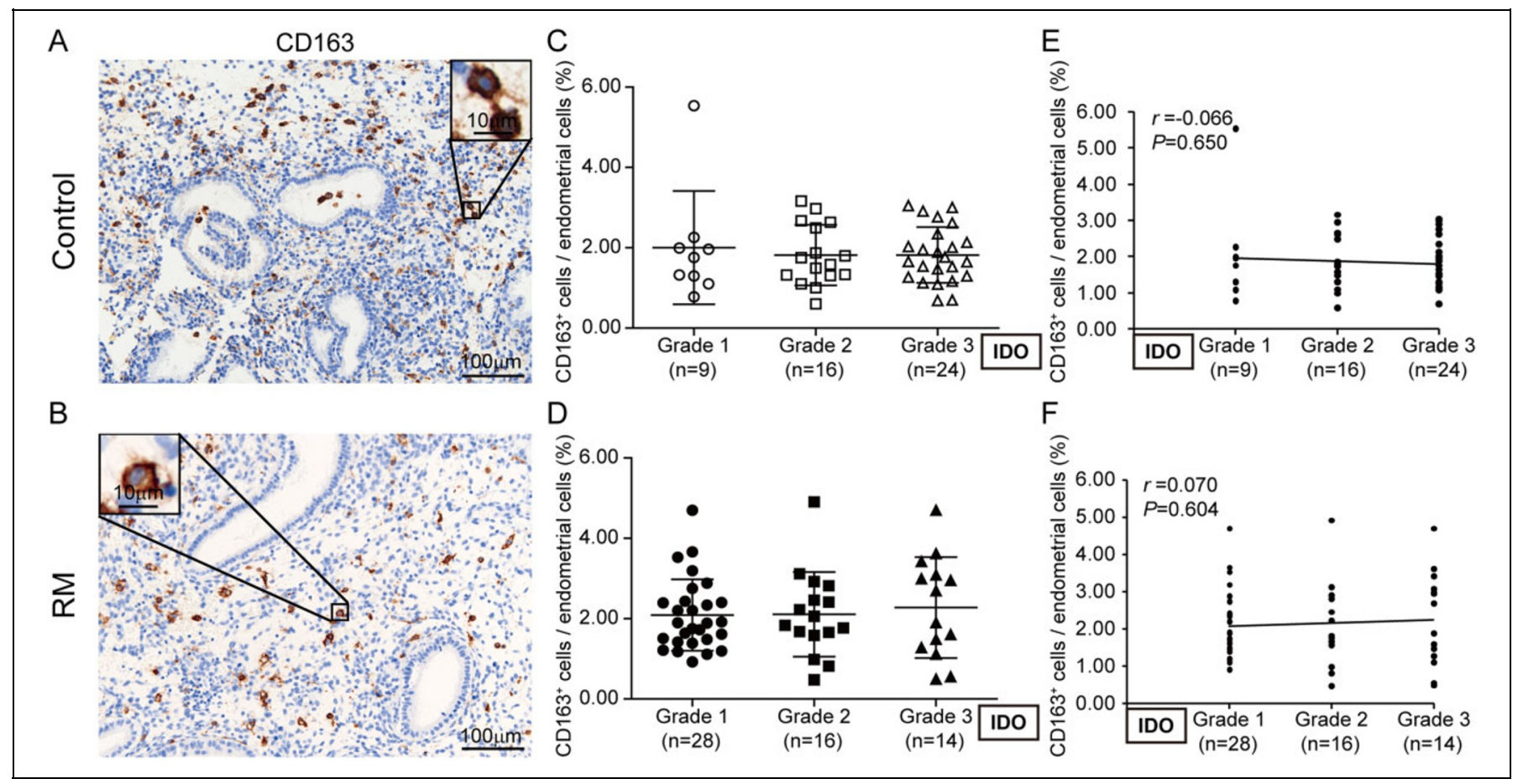

Figure 4. Immunohistochemical examination of CDI63 in endometrium and the relationship analysis between IDO and CDI63 ${ }^{+} \mathrm{M} 2$-type macrophages. Representative images taken at a magnification of $200 \times$ field of endometrial CDI63 ${ }^{+}$M2-type macrophages in control women (A) and RM patients (B). Scale bars $=100 \mu \mathrm{m}$, with insets $=10 \mu \mathrm{m}$. Summary graph of statistical dot plot (mean [SD]) showing the percentages of endometrial CDI63 among 3 subgroups in control group (grade I, $n=9$; grade 2, n= I6; grade 3, $n=24 ; C$ ) and RM group (grade I, $n=28$; grade 2, $n=16$; grade 3, $n=14 ; D)$. One-way ANOVA test. Numerical correlation between the percentage of CDI63 ${ }^{+} M 2$-type macrophages and the level of IDO expression in healthy fertile controls $(E)$ and patients with RM (F). Pearson correlation, $r$ and $P$ values as indicated. IDO indicates indoleamine 2, 3-dioxygenase; NK, natural killer; RM, recurrent miscarriage.

degree of IDO expression, IFN- $\gamma$ could upregulate the expression of M1 markers such as HLA-DR and CCR7 and induce monocytes to M1-polarization. ${ }^{32,28}$ However, it has also been revealed that the ectopic IDO could increase the expression of M2-type markers such as IL-10 and CXCR-4, associated with the decrease in M1-type markers. ${ }^{32}$ Our study demonstrated a stable expression of endometrial CD163 with elevated IDO expression from both controls and RM patients. Taken together, the results recapitulated that IDO may coordinate with the number of endometrial Tregs and NK cells, rather than macrophages.

In conclusion, our study demonstrated the characteristic distribution of IDO protein in endometrium. Significantly decreased level of IDO expression was observed in RM samples, suggesting IDO may contribute to maternal tolerance to semiallogeneic fetus in normal pregnancy. Additionally, our data showed that the percentage of Foxp $3^{+}$Tregs was significantly correlated with the level of IDO expression in the control group, suggesting that IDO may be involved in the maintenance of maternal tolerance through the regulation of Foxp $3^{+}$Treg. However, this correlation was impaired in patients with RM. It should be taken into account that IDO expression may not exactly reflect the enzyme activity. To clarify the exact role of IDO in immune regulation, further studies should be performed. Taken together, our findings may provide a basis for further research into the role of IDO in immunoregulation of human pregnancy and may open up an attractive drug target for IDO-related RM therapy.

\section{Authors' Note}

Hongxia Wei and Su Liu contributed equally to this work

\section{Acknowledgments}

The authors would like to thank the staff at the Fertility Center at Shenzhen Zhongshan Urology Hospital for recruiting patients and helping with samples.

\section{Declaration of Conflicting Interests}

The author(s) declared no potential conflicts of interest with respect to the research, authorship, and/or publication of this article.

\section{Funding}

The author(s) disclosed receipt of the following financial support for the research, authorship, and/or publication of this article: This work was supported by Science and Technology Project of Health and Family Planning Commission of Shenzhen Municipality (SZBC2018003), Basic Research Program of Shenzhen (JCYJ20170307140647669), specific funding from Clinical Medical Research of Chinese Medical Association-Reproductive Medicine Clinical Research and Development Youth Programs (17020300699) and Sanming Project of Medicine in Shenzhen (SZSM201502035). 


\section{References}

1. Ford HB, Schust DJ. Recurrent pregnancy loss: etiology, diagnosis, and therapy. Rev Obstet Gynecol. 2009;2(2):76-83.

2. Li TC, Makris M, Tomsu M, Tuckerman E, Laird S. Recurrent miscarriage: aetiology, management and prognosis. Hum Reprod Update. 2002;8(5):463-481.

3. Macklon NS, Brosens JJ. The human endometrium as a sensor of embryo quality. Biol Reprod. 2014;91(4):98.

4. Evans J, Salamonsen LA, Winship A, et al. Fertile ground: human endometrial programming and lessons in health and disease. Nat Rev Endocrinol. 2016;12(11):654-667.

5. Mekinian A, Cohen J, Kayem G, et al. [Unexplained recurrent early miscarriages: Role of immunomodulation?]. Rev Med Interne. 2017;38(4):264-268.

6. Katz JB, Muller AJ, Prendergast GC. Indoleamine 2,3dioxygenase in T-cell tolerance and tumoral immune escape. Immunol Rev. 2008;222:206-221.

7. Munn DH, Zhou M, Attwood JT, et al. Prevention of allogeneic fetal rejection by tryptophan catabolism. Science. 1998; 281(5380):1191-1193.

8. Mellor AL, Munn DH. IDO expression by dendritic cells: tolerance and tryptophan catabolism. Nat Rev Immunol. 2004;4(10): 762-774.

9. Munn DH, Mellor AL. Indoleamine 2,3-dioxygenase and tumorinduced tolerance. J Clin Invest. 2007;117(5):1147-1154.

10. Ban Y, Chang Y, Dong B, Kong B, Qu X. Indoleamine 2,3-dioxygenase levels at the normal and recurrent spontaneous abortion fetal-maternal interface. J Int Med Res. 2013;41(4): 1135-1149.

11. Liu X, Liu Y, Ding M, Wang X. Reduced expression of indoleamine 2,3-dioxygenase participates in pathogenesis of preeclampsia via regulatory T cells. Mol Med Rep. 2011;4(1):53-58.

12. Badawy AA, Namboodiri AM, Moffett JR. The end of the road for the tryptophan depletion concept in pregnancy and infection. Clin Sci. 2016;130(15):1327-1333.

13. Ebina Y, Shimada S, Deguchi M, Maesawa Y, Iijima N, Yamada $\mathrm{H}$. Divergence of helper, cytotoxic, and regulatory $\mathrm{T}$ cells in the decidua from miscarriage. Am J Reprod Immunol. 2016;76(3): 199-204.

14. Quan X, Yang X. Correlation between unexplained recurrent spontaneous abortion with $\mathrm{CD} 4(+) \mathrm{CD} 25(+)$ regulatory $\mathrm{T}$-cell and killer cell immunoglobulin-like receptor levels. Exp Ther Med. 2017;14(2):1459-1462.

15. Fallarino F, Grohmann U, You S, et al. The combined effects of tryptophan starvation and tryptophan catabolites down-regulate $\mathrm{T}$ cell receptor zeta-chain and induce a regulatory phenotype in naive T cells. J Immunol. 2006;176(11):6752-6761.

16. Sharma MD, Baban B, Chandler P, et al. Plasmacytoid dendritic cells from mouse tumor-draining lymph nodes directly activate mature Tregs via indoleamine 2,3-dioxygenase. J Clin Invest. 2007;117(9):2570-2582.

17. Chen W, Liang X, Peterson AJ, Munn DH, Blazar BR. The indoleamine 2,3-dioxygenase pathway is essential for human plasmacytoid dendritic cell-induced adaptive $\mathrm{T}$ regulatory cell generation. J Immunol. 2008;181(8):5396-5404.
18. Manches O, Munn D, Fallahi A, et al. HIV-activated human plasmacytoid DCs induce Tregs through an indoleamine 2,3-dioxygenase-dependent mechanism. J Clin Invest. 2008; 118(10):3431-3439.

19. van der Marel AP, Samsom JN, Greuter M, et al. Blockade of IDO inhibits nasal tolerance induction. J Immunol. 2007;179(2): 894-900.

20. Matteoli G, Mazzini E, Iliev ID, et al. Gut CD103+ dendritic cells express indoleamine 2,3-dioxygenase which influences $\mathrm{T}$ regulatory/T effector cell balance and oral tolerance induction. Gut. 2010;59(5):595-604.

21. Vivier E, Tomasello E, Baratin M, Walzer T, Ugolini S. Functions of natural killer cells. Nat Immunol. 2008;9(5):503-510.

22. Della Chiesa M, Carlomagno S, Frumento G, et al. The tryptophan catabolite L-kynurenine inhibits the surface expression of NKp46- and NKG2D-activating receptors and regulates NK-cell function. Blood. 2006;108(13):4118-4125.

23. Gaynor LM, Colucci F. Uterine natural killer cells: functional distinctions and influence on pregnancy in humans and mice. Front Immunol. 2017;8:467.

24. Kuon RJ, Weber M, Heger J, et al. Uterine natural killer cells in patients with idiopathic recurrent miscarriage. Am J Reprod Immunol. 2017;78(4):e12721.

25. Sato N, Saga Y, Mizukami H, et al. Downregulation of indoleamine-2,3-dioxygenase in cervical cancer cells suppresses tumor growth by promoting natural killer cell accumulation. Oncol Rep. 2012;28(5):1574-1578.

26. Frumento G, Rotondo R, Tonetti M, Damonte G, Benatti U, Ferrara GB. Tryptophan-derived catabolites are responsible for inhibition of $\mathrm{T}$ and natural killer cell proliferation induced by indoleamine 2,3-dioxygenase. J Exp Med. 2002;196(4):459-468.

27. Mege JL, Mehraj V, Capo C. Macrophage polarization and bacterial infections. Curr Opin Infect Dis. 2011;24(3):230-234.

28. Benoit M, Desnues B, Mege JL. Macrophage polarization in bacterial infections. J Immunol. 2008;181(6):3733-3739.

29. Gronlund J, Vitved L, Lausen M, Skjodt K, Holmskov U. Cloning of a novel scavenger receptor cysteine-rich type I transmembrane molecule (M160) expressed by human macrophages. J Immunol. 2000;165(11):6406-6415.

30. Buechler C, Ritter M, Orso E, Langmann T, Klucken J, Schmitz G. Regulation of scavenger receptor CD163 expression in human monocytes and macrophages by pro- and antiinflammatory stimuli. J Leukocyte Biol. 2000;67(1):97-103.

31. Komohara Y, Hirahara J, Horikawa T, et al. AM-3K, an antimacrophage antibody, recognizes CD163, a molecule associated with an anti-inflammatory macrophage phenotype. J Histochem Cytochem: official journal of the Histochemistry Society. 2006; 54(7):763-771.

32. Wang XF, Wang HS, Wang $\mathrm{H}$, et al. The role of indoleamine 2,3-dioxygenase (IDO) in immune tolerance: focus on macrophage polarization of THP-1 cells. Cell Immunol. 2014; 289(1-2):42-48.

33. Iwahashi N, Yamamoto M, Nanjo S, Toujima S, Minami S, Ino K. Downregulation of indoleamine 2, 3-dioxygenase expression in the villous stromal endothelial cells of placentas with preeclampsia. J Reprod Immunol. 2017;119:54-60. 
34. Sedlmayr P, Blaschitz A, Wintersteiger R, et al. Localization of indoleamine 2,3-dioxygenase in human female reproductive organs and the placenta. Mol Hum Reprod. 2002;8(4): 385-391.

35. Ligam P, Manuelpillai U, Wallace EM, Walker D. Localisation of indoleamine 2,3-dioxygenase and kynurenine hydroxylase in the human placenta and decidua: implications for role of the kynurenine pathway in pregnancy. Placenta. 2005;26(6):498-504.

36. Sandra O, Mansouri-Attia N, Lea RG. Novel aspects of endometrial function: a biological sensor of embryo quality and driver of pregnancy success. Reprod Fertil Dev. 2011;24(1):68-79.

37. Chang RQ, Li DJ, Li MQ. The role of indoleamine-2,3dioxygenase in normal and pathological pregnancies. Am J Reprod Immunol. 2017;79(4):e12786.

38. Kudo Y, Boyd CA, Spyropoulou I, et al. Indoleamine 2,3dioxygenase: distribution and function in the developing human placenta. J Reprod Immunol. 2004;61(2):87-98.

39. Miwa N, Hayakawa S, Miyazaki S, et al. IDO expression on decidual and peripheral blood dendritic cells and monocytes/ macrophages after treatment with CTLA-4 or interferon-gamma increase in normal pregnancy but decrease in spontaneous abortion. Mol Hum Reprod. 2005;11(12):865-870.

40. Diao LH, Li GG, Zhu YC, et al. Human chorionic gonadotropin potentially affects pregnancy outcome in women with recurrent implantation failure by regulating the homing preference of regulatory T cells. Am J Reprod Immunol. 2017; 77(3):e12618.

41. Leavy O. Tolerance: induced T(Reg) cells evolved to protect the fetus. Nat Rev Immunol. 2012;12(8):554-555.

42. Munn DH, Mellor AL. Indoleamine 2,3 dioxygenase and metabolic control of immune responses. Trends Immunol. 2013;34(3): 137-143.

43. Sundrud MS, Koralov SB, Feuerer M, et al. Halofuginone inhibits TH17 cell differentiation by activating the amino acid starvation response. Science. 2009;324(5932):1334-1338.
44. Keller TL, Zocco D, Sundrud MS, et al. Halofuginone and other febrifugine derivatives inhibit prolyl-tRNA synthetase. Nat Chem Biol. 2012;8(3):311-317.

45. Nguyen NT, Kimura A, Nakahama T, et al. Aryl hydrocarbon receptor negatively regulates dendritic cell immunogenicity via a kynurenine-dependent mechanism. Proc Natl Acad Sci U S A. 2010;107(46):19961-19966.

46. Mezrich JD, Fechner JH, Zhang X, Johnson BP, Burlingham WJ, Bradfield CA. An interaction between kynurenine and the aryl hydrocarbon receptor can generate regulatory T cells. J Immunol. 2010;185(6):3190-3198.

47. Chung DJ, Rossi M, Romano E, et al. Indoleamine 2,3dioxygenase-expressing mature human monocyte-derived dendritic cells expand potent autologous regulatory T cells. Blood. 2009;114(3):555-563.

48. Russell P, Sacks G, Tremellen K, Gee A. The distribution of immune cells and macrophages in the endometrium of women with recurrent reproductive failure. III: further observations and reference ranges. Pathology. 2013;45(4):393-401.

49. Horowitz A, Strauss-Albee DM, Leipold M, et al. Genetic and environmental determinants of human NK cell diversity revealed by mass cytometry. Sci Transl Med. 2013;5(208):208ra145.

50. Gordon S. Alternative activation of macrophages. Nat Rev Immunol. 2003;3(1):23-35.

51. Mantovani A, Sica A, Sozzani S, Allavena P, Vecchi A, Locati M. The chemokine system in diverse forms of macrophage activation and polarization. Trends Immunol. 2004;25(12):677-686.

52. Watkins SK, Egilmez NK, Suttles J, Stout RD. IL-12 rapidly alters the functional profile of tumor-associated and tumorinfiltrating macrophages in vitro and in vivo. J Immunol. 2007; 178(3):1357-1362.

53. Tjiu JW, Chen JS, Shun CT, et al. Tumor-associated macrophageinduced invasion and angiogenesis of human basal cell carcinoma cells by cyclooxygenase-2 induction. J Invest Dermatol. 2009; 129(4):1016-1025. 